\title{
Spectral Analysis of New X-ray Outbursts from the SFXT AX J1845.0-0433
}

\section{Maximiliane Goossens*1, Angela Bazzano ${ }^{2}$, Antony Bird ${ }^{1}$, Sebastian Drave ${ }^{1}$, Adam Hill $^{1,3}$, Vito Sguera ${ }^{4}$, Lara Sidoli ${ }^{5}$}

${ }^{1}$ School of Physics and Astronomy, University of Southampton, University Road, Southampton, SO17 1BJ, UK

${ }^{2}$ IAPS-INAF, Instituto di Astrofisica Spaziale e Fisica Cosmica, Via Fosso del Cavaliere 100, I-00133 Roma, Italy

${ }^{3}$ W. W. Hansen Experimental Physics Laboratory, Kavli Institute for Particle Astrophysics and Cosmology, Department of Physics and SLAC National Accelerator Laboratory, Stanford University, Stanford, CA 94305, USA

${ }^{4}$ INAF-IASF, Instituto di Astrofisica Spaziale e Fisica Cosmica, Via Gobetti 101, I-40129 Bologna, Italy

${ }^{5}$ INAF-IASF, Instituto di Astrofisica Spaziale e Fisica Cosmica, Via E. Bassini 15, I-20133

Milano, Italy

E-mail: m.e.goossensesoton.ac.uk

\begin{abstract}
AX J1845.0-0433 was first discovered during an ASCA observation in 1993. Later it was found that because of their similar positioning, IGR J18450-0435 and AX J18450-0433 are likely to be the same object. The system exhibits X-ray transient behaviour and a supergiant star was found to be its optical counterpart. This suggests that AX J1845.0-0433 is a member of the high mass X-ray binary (HMXB) subclass of supergiant fast X-ray transients (SFXTs). Here we present a detailed investigation of the outburst history of the source, identifying 14 new flares, as well as a spectral study of the brightest new outbursts.
\end{abstract}

An INTEGRAL view of the high-energy sky (the first 10 years) - 9th INTEGRAL Workshop and celebration of the 10th anniversary of the launch

15-19 October 2012

Bibliotheque Nationale de France, Paris, France

\footnotetext{
* Speaker.
} 


\section{Introduction}

AX J1845.0-0433 was first discovered in the Scutum arm region during an ASCA observation in 1993 at a position of $\operatorname{RA}(2000)=18^{h} 45^{m} 2^{s}, \operatorname{Dec}(2000)=-4^{\circ} 33^{\prime} 31^{\prime \prime}$. It was observed that the source, initially in a quiescent state $\left(0.7-10 \mathrm{keV}\right.$ flux of $\left.3 \times 10^{-12} \mathrm{erg} \mathrm{cm}^{-2} \mathrm{~s}^{-1}\right)$, flared up on a time scale of less than an hour until it reached a peak flux of $\sim 10^{-9} \mathrm{erg} \mathrm{cm}^{-2} \mathrm{~s}^{-1}$ (Yamauchi et al., 1995). The flat X-ray spectra obtained for both the quiescent as well as the flare states both resembled those of transient X-ray binary pulsars, which led to conclude that AX J1845.0-0433 is a massive binary system consisting of a neutron star with either a Be or a supergiant companion. The companion star was later identified by Coe et al. (1996). Optical as well as infrared measurements of the two brightest objects in the ASCA error circle ( 1 arcmin radius) were taken and consequently the counterpart was determined to be an O9.5I supergiant star with an estimated distance of 3.6 kpc.

IGR J18450-0435 was first discovered by INTEGRAL (Molkov et al., 2004) and later Halpern et al. (2006) noted that due to similar positioning AX J1845.0-0433 and IGR J18450-0435 are likely to be the same object. This was confirmed by Sguera et al. (2007) announcing that the optical counterpart of AX J1845.0-0433 is located 0.5' from the ISGRI position of IGR J18450-0435.

The fast X-ray transient behaviour as well as its association with a supergiant star classifies AX J1845.0-0433 as a member of the high mass X-ray binary (HMXB) subclass of supergiant fast X-ray transients (SFXTs) (Negueruela et al, 2005; Sguera et al., 2005). SFXTs are occasionally observed in X-ray quiescence $\left(L_{x}<10^{32} \mathrm{ergs}^{-1}\right)$ but more frequently during a low X-ray state with $L_{x}=10^{33}-10^{34} \mathrm{ergs}^{-1}$. Rarely, however, fast X-ray transient activity can be observed from these systems. Often this lasts less than a day and is characterised by short flares lasting only a few tens of minutes with peak luminosities of $\sim 10^{36} \mathrm{ergs}^{-1}$.

The two most recent outburst activities from AX J1845.0-0433 were reported by Sguera et al. (2007) and Ducci et al. (2010). The ones reported by Sguera et al. (2007) were the first ones to be announced since the source's discovery in 1993.

Here we report the observation of two new outbursts of AX J1845.0-0433 occurring within a few days of each other in March 2010.

\section{Data Set and Analysis}

The INTEGRAL gamma-ray observatory consists of three co-aligned coded masks telescopes: the X-ray monitor (JEM-X) (Lund et al., 2003), the spectrometer (SPI) (Vedrenne et al., 2003) and the imager (IBIS) (Ubertini et al., 2003). In this paper only results from IBIS/ISGRI are presented. INTEGRAL observations are divided into science windows with a duration of $\sim 2000$ s (Jensen et al., 2003). Using the INTEGRAL offline analysis software, OSA v.9 (Goldwurm et al., 2003; Courvoisier et al., 2003), we analysed all the available IBIS/ISGRI data covering the range from $10^{\text {th }}$ March 2003 to $29^{\text {th }}$ September 2010 (MJD range 52708-55704) in the 18-60 keV energy band. Moreover, 16 further science windows of public Galactic Plane Scan (GPS2) data were added ranging from $15^{\text {th }}$ May 2011 to $10^{\text {th }}$ February 2012 so as to include a greater time span in the analysis. 


\section{Searching for New Outbursts}

A comprehensive search for new outbursts was carried out on the 18-60 keV IBIS/ISGRI light curve. This was done by using a significance test where a "sliding window" was utilised to calculate the weighted means and errors of all the science windows within 0.2 days ( $\sim 5$ hours) of each other considering every data point in the light curve. Science windows within that time frame were considered to be part of the same outburst activity so that the significance of the individual flares could be computed.

In order to assess the maximum significance that may be generated by statistical fluctuations in the data, a distribution of the significance values detected within the inverted light curve was derived. $99.9 \%$ of the random fluctuations generated significances below $5.8 \sigma$ and therefore, $5.8 \sigma$ was taken as the $99.9 \%$ confidence limit of an outburst in the real light curve being of a physical origin. Applying this limit to the events detected in the true light curve revealed seventeen outburst events. Three of those are the ones already discovered in IBIS (numbers 2, 5 and 6 in Table 1) resulting in another fourteen new flares. Out of these, only the ones where a meaningful spectral analysis could be performed, i.e. outburst events of at least $8 \sigma$ significance, are given in Table 1 , listing the date on which they occurred, the energy band they were detected in, their peak flux and peak luminosity values as well as their photon indices. In this table outbursts 7 and 8 represent the newly discovered flares.

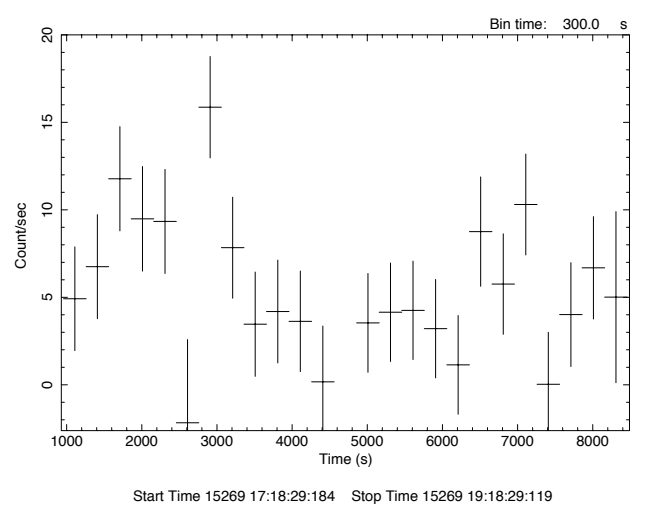

Figure 1: IBIS/ISGRI light curve of AX J1845.00433 (18-60 keV) during the observation on 14th March 2010 (outburst no. 7 in Table 1).

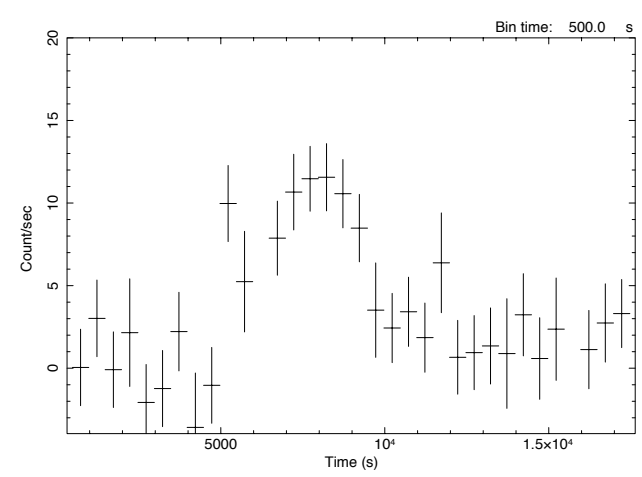

Start Time 15275 17:12:03:184 Stop Time 15275 21:47:03:057

Figure 2: IBIS/ISGRI light curve of AX J1845.0$0433(18-60 \mathrm{keV})$ during the observation on 20th March 2010 (outburst no. 8 in Table 1).

\subsection{Results on the first outburst activity}

Figure 1 displays the $18-60 \mathrm{keV}$ light curve of AX J1845.0-0433 during the outburst on $14^{\text {th }}$ March 2010. It can be seen that the source underwent two short flares lasting $\sim 20$ minutes each starting at about 17:18 UTC. The first one had a peak flux of $3.4 \times 10^{-10} \mathrm{erg} \mathrm{cm}^{-2} \mathrm{~s}^{-1}$ and the peak luminosity was calculated as $5.2 \times 10^{35} \mathrm{ergs}^{-1}(9.7 \sigma)$. The second flare was somewhat fainter and observed about an hour after the first one. A spectrum for the selected science windows was extracted and any data points below $20 \mathrm{keV}$ were excluded as the ISGRI response is not well 


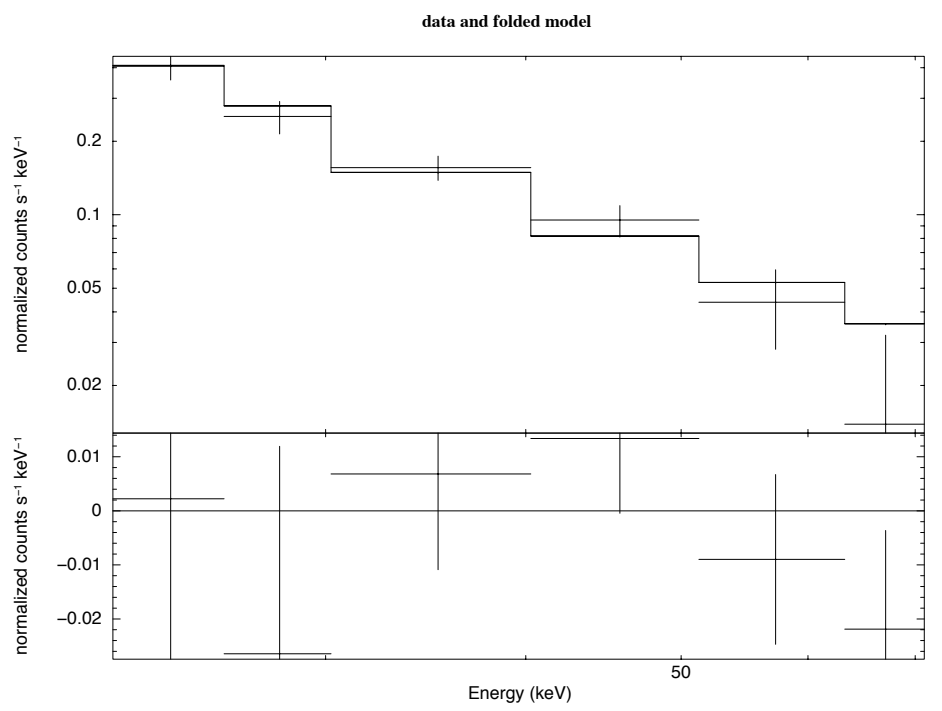

Figure 3: Top: Combined spectrum (20-80 keV) of AX J1845.0-0433 during the two new outbursts. Bottom: A plot of the residuals.

modelled below $20 \mathrm{keV}$. Finally, a power law was fitted to the spectrum $\left(\chi_{r}^{2}=0.733,8\right.$ d.o.f $)$ with $\Gamma=2.53_{-0.3}^{+0.3}$.

\subsection{Results on the second outburst activity}

Figure 2 displays the 18-60keV light curve of AX J1845.0-0433 during the outburst on $20^{\text {th }}$ March 2010. It can be seen that the source underwent one major flare lasting about 1.5 hours starting at $\sim$ 17:12 UTC. The outburst had a peak flux of $\sim 4.4 \times 10^{-10} \mathrm{erg} \mathrm{cm}^{-2} \mathrm{~s}^{-1}$ and the peak luminosity was calculated to be $6.9 \times 10^{35} \mathrm{erg} \mathrm{s}^{-1}(10.8 \sigma)$ as can be seen in Table 1. A spectrum for the selected science windows was extracted and any data points below $20 \mathrm{keV}$ were excluded before a power law $\left(\chi_{r}^{2}=0.687,8\right.$ d.o.f. $)$ was fitted to it with $\Gamma=2.84_{-0.3}^{+0.3}$.

Comparing the fitting parameters of the individual spectra it could be noted that they are consistent within errors so that a combined spectrum could be produced. The spectrum was then filtered such that any data points outside the $20-80 \mathrm{keV}$ range were excluded due to their poor quality. Finally, it was then fitted with a normal power law $\left(\chi_{r}^{2}=0.831\right.$, 4 d.o.f $)$ with $\Gamma=2.49_{-0.2}^{+0.2}$. The resulting fitted spectrum can be seen in Figure 3 together with a residuals plot.

\section{Conclusions}

Carrying out a significance test on the 18-60 keV IBIS/ISGRI light curve of the SFXT AX J1845.0-0433 resulted in fourteen new outbursts being observed out of which two were above a calculated significance limit required for a more detailed analysis. Both of them are of similar duration and their corresponding peak flux values and consequently their intensities are very alike. A spectral analysis of each of the two outburst activities revealed comparable parameters when fitting 
a normal power law so that a combined spectrum for both outbursts could be produced. The resulting fitting parameters from the combined spectrum agree with findings from other outbursts from this source e.g. Sguera et al. (2007). There are not many confirmed outbursts for AX J1845.0-0433 resulting in a low recurrence rate and no significantly brighter burst has been identified compared to what can be observed from some other SFXTs e.g. SAX J1818.6-1703 (Bird et al., 2008). All outbursts known to date can be seen in Table 1, which puts the new flares into context with the already observed ones. Overall, AX J1845.0-0433 seems to be a standard intermediate SFXT.

Table 1: Summary of INTEGRAL, Swift/BAT and ASCA observations of outbursts of AX J1845.0-0433 (Sguera et al., 2007; Ducci et al., 2010). Numbers 7-8 are the newly discovered outbursts above $8 \sigma$ significance. Fourteen more outbursts were detected but are not included in this table.

\begin{tabular}{|c|c|c|c|c|c|c|c|}
\hline No. & Observation & Date & $\begin{array}{c}\text { Energy Band } \\
(\mathrm{keV})\end{array}$ & $\begin{array}{c}\text { Peak Flux } \\
\left(\operatorname{erg~cm}^{-2} \mathrm{~s}^{-1}\right)\end{array}$ & $\begin{array}{c}\text { Peak Luminosity } \\
\left(\mathrm{ergs}^{-1}\right)\end{array}$ & $\begin{array}{l}\text { Photon Index } \\
\text { (Power Law) }\end{array}$ & $\begin{array}{c}\mathrm{N}_{\mathrm{H}} \\
\left(\mathrm{cm}^{-2}\right)\end{array}$ \\
\hline 1 & $A S C A$ & 18 Oct. 1993 & $0.7-10$ & $1.0 \times 10^{-9}$ & $1.5 \times 10^{36}$ & $1.0_{-0.07}^{+0.07}$ & $3.6 \pm 0.3 \times 10^{22}$ \\
\hline 2 & INTEGRAL/ISGRI & 28 Apr. 2005 & $20-40$ & $4.5 \times 10^{-10}$ & $7.0 \times 10^{35}$ & $2.5_{-0.5}^{+0.6}$ & \\
\hline 3 & Swift/XRT & 11 Nov. 2005 & $0.2-10$ & $2.3 \times 10^{-10}$ & $3.6 \times 10^{35}$ & $0.75_{-0.1}^{+0.1}$ & $1.6 \pm 0.18 \times 10^{22}$ \\
\hline 4 & Swift/XRT & 05 Mar. 2006 & $0.2-10$ & $1.1 \times 10^{-10}$ & $2.0 \times 10^{35}$ & $0.85_{-0.3}^{+0.3}$ & $2.3 \pm 0.7 \times 10^{22}$ \\
\hline 5 & INTEGRAL/ISGRI & 20 Apr. 2006 & $20-40$ & $6.0 \times 10^{-10}$ & $9.3 \times 10^{35}$ & $2.9_{-0.7}^{+0.9}$ & \\
\hline 6 & INTEGRAL/ISGRI & 03 Sept. 2006 & $18-60$ & $2.5 \times 10^{-10}$ & $3.9 \times 10^{35}$ & $2.62_{-0.4}^{+0.4}$ & \\
\hline 7 & INTEGRAL/ISGRI & 14 Mar. 2010 & $18-60$ & $3.4 \times 10^{-10}$ & $5.2 \times 10^{35}$ & $2.53_{-0.3}^{+0.3}$ & \\
\hline 8 & INTEGRAL/ISGRI & 20 Mar. 2010 & $18-60$ & $4.4 \times 10^{-10}$ & $6.9 \times 10^{35}$ & $2.84_{-0.3}^{+0.3}$ & \\
\hline
\end{tabular}

\section{References}

A. J. Bird, A. Bazzano, A. B. Hill et al., Discovery of a 30-d period in the supergiant fast X-ray transient SAX J1818.6-1703, 2008, MNRAS, 393, L11

M. Coe, J. Fabregat, I. Negueruela et al., Discovery of the optical counterpart to the ASCA transient AX 1845.0-0433,1996, MNRAS, 281, 333

T. J. Courvoisier, R. Walter, V. Beckmann et al., The INTEGRAL Science Data Centre (ISDC), 2003, A\&A, 411, L53

L. Ducci, L. Sidoli, A. Paizis, INTEGRAL results on supergiant fast $X$-ray transients and accretion mechanisms interpretation: ionization effect and formation of transient accretion discs, 2010, MNRAS, 408, 1540

A. Goldwurm, P. David, L. Foschini et al., The INTEGRAL/IBIS scientific data analysis, 2003, A\&A, 411, L223

J. P. Halpern, E. V. Gotthelf, IGR J18450-0435=AX J1845.0-0433,2006, ATEL, 692

P. L. Jensen, K. Clausen, C. Cassi et al., The INTEGRAL spacecraft - in-orbit performance, 2003, A\&A, 411, L7 
N. Lund, C. Butz-Jørgentsen, N. J. Westergaard et al., JEM-X: The X-ray monitor aboard INTEGRAL, 2003, A\&A, 411, L231

S. V. Molkov, A. M. Cherepashchuk, A. A. Lutovinov et al., A hard X-ray survey of the Sagittarius Arm tangent with the IBIS telescope of the INTEGRAL observatory: A catalog of sources, 2004, A\&A, 30, L534

I. Negueruela, D. M. Smith, P. Reig et al., Supergiant Fast X-ray Transients: A New Class of High Mass X-ray Binaries Unveiled by INTEGRAL, 2005, ESA SP-604, 165

V. Sguera, E. J. Barlow, A. J. Bird et al., INTEGRAL observations of recurrent fast X-ray transient sources, 2005, A\&A, 444, L221

V. Sguera, A. J. Bird, A. J. Dean et al., INTEGRAL and Swift observations of the supergiant fast $X$-ray transient AX J1845.0-0433=IGR J18450-0435, 2007, A\&A, 462, 696

P. Ubertini, F. Lebrun, G. Di Cocco et al., IBIS: The Imager on-board INTEGRAL, 2003, A\&A, 411, L131

G. Vedrenne, J. Roques, V. Schönfelder et al., SPI: The spectrometer aboard INTEGRAL, 2003, A\&A, 411, L63

S. Yamauchi, T. Aoki, K. Hayashida et al., New Transient X-Ray Source in the Scutum Region Discovered with ASCA, 1995, PASJ, 47, 189 